\title{
IDENTIFYING AND UNDERSTANDING OPIOID USE DISORDER IN CLINICAL NOTES
}

\author{
T. Elizabeth Workman ${ }^{1}$, Joel Kupersmith ${ }^{2}$, Joseph L. Goulet ${ }^{3}$, Christopher Spevak ${ }^{4}$, \\ Cynthia Brandt ${ }^{5}$, Friedhelm Sandbrink ${ }^{6}$, Marc R. Blackman ${ }^{7}$, Nawar M. Shara ${ }^{8}$ \\ and Qing Zeng-Treitler ${ }^{1}$ \\ ${ }^{I}$ Biomedical Informatics Center, The George Washington University, Washington, D.C., U.S.A \\ ${ }^{2}$ Veterans Initiatives, Georgetown University, Washington, D.C., U.S.A \\ ${ }^{3}$ VA Connecticut Healthcare System, West Haven, CT, U.S.A \\ ${ }^{4}$ Prescription Medication Misuse Program, Georgetown University, Washington, D.C., U.S.A \\ ${ }^{5}$ Biostatistics, Yale University, New Haven, CT, U.S.A \\ ${ }^{6}$ Pain Management, VA Medical Center, Washington, D.C., U.S.A \\ ${ }^{7}$ Medicine and Rehabilitation Medicine, Georgetown University, Washington, D.C., U.S.A \\ ${ }^{8}$ Biostatistics and Biomedical Informatics, MedStar Health Research, Hyattsville, MD, U.S.A
}

\begin{abstract}
Opioid use, abuse and misuse afflicts many populations, including Veterans. The objective of this ten-year retrospective study was to identify documentation of potential opioid abuse, both treated and untreated, in clinical notes, by developing and applying a natural language processing tool to a corpus of clinical notes documenting the healthcare of U.S. Veterans. To better understand the issue of opioid abuse among Veterans, we also extracted descriptive data on prescription counts, patient demographics, and diagnoses. The natural language processing tool we developed achieved F1 scores of $88 \%$ and $91 \%$ in identifying opioid abuse with treatment, and without treatment, respectively, among U.S. Veterans receiving healthcare in the Baltimore, Maryland and Washington DC VA service regions. This resulted in identifying 809 additional patients experiencing opioid abuse. The descriptive data give insight by elucidating trends that enhance understanding of opioid abuse among Veterans receiving healthcare in these service regions, and suggest future research.
\end{abstract}

\section{KEYWORDS}

Opioid Abuse Disorder, Machine Learning, U.S. Veterans

\section{INTRODUCTION}

The misuse of prescribed opioids is a global problem affecting numerous populations (Martins et al., 2015). In 2017, there were 47,600 deaths the United States attributed to opioid overdoses (Scholl et al., 2018). The Diagnostic and Statistical Manual of Mental Disorders, $5^{\text {th }}$ Edition (Association, 2013) defines Opioid Abuse Disorder (OUD) as a problematic pattern of opioid use leading to clinically significant impairment or distress. Its characteristics include clinical heterogeneity (Kendler et al., 2013), having diverse risk factors, including past substance abuse, age, untreated psychiatric disorders, family situation, traumatic brain injury, and pain (Webster, 2017, Cottler et al., 2011, Edlund et al., 2007). Variance in geographic prescribing patterns affects the complexity of OUD (Garcia et al., 2019). Facets involving gender and race also present parallel considerations in studying OUD, particularly in pain management. African Americans and other ethnic minorities are consistently under-accessed and undertreated for pain (Green et al., 2003). Women experiencing chronic pain and opioid abuse tend to report more pain-related impairment and psychiatric co-morbidity, while their male counterparts report more aberrant behaviors (Manubay et al., 2015). Women may also demonstrate lower pain thresholds, and higher pain ratings than men (Chartoff and Mavrikaki, 2015). Opioid abuse also affects Veterans of the United States Military, for whom male sex, young age, and mental health disorders are notable risk factors (Edlund et al., 2007). Several policies and practice guidelines have attempted to address the problem of opioid abuse among U.S. Veterans, including the Opioid Safety Initiative (OSI) in 2013 and the stratification tool for opioid risk management (STORM) in 2018 (Management, Gellad et al., 2017, Lin et al., 2017, Wentling, 2018). 
In electronic medical records (EMRs), opioid use, abuse, and misuse is recorded as structured data, in the form of diagnostic codes, or in the unstructured data found in clinical notes. While clinicians often record information as structured data, sometimes some information may be found only in a patient's notes (Moss et al., 2007). Using only ICD codes in structured data as an information source may underestimate the incidences of opioid abuse and result in errors in subsequent analyses. It is therefore desirable to identify clinical notes specifically addressing opioid abuse along with information addressing treatment. This can potentially be facilitated through natural language processing (NLP) and machine learning by preparing the notes using standard NLP techniques such as ngram assessment, where an ngram is an instance of a single word (unigram), or instances of adjacent multiple words in a corpus. This type of assessment includes capturing instances of two adjacent words (bigrams) throughout a corpus of clinical notes, and using the counts of each bigram as features for each clinical note, in training a machine learning model.

Other studies have applied machine learning applications to gain insight into opioid use, abuse and misuse. Several of these focus on social media content (Sarker et al., 2019b, Sarker et al., 2019a) or structured EMR data (Hastings et al., 2020, Workman et al., 2019b), or structured data and clinical notes, with NLP (Lingeman et al., 2017, Sharma et al., 2020). Sharma et al. identified a random case-control cohort of 1000 patients with or without ICD-9/ICD-10 OUD related diagnosis or positive urine drug screen for opioids, subjected to chart review (Sharma et al., 2020). They experimented with both ngram and UMLS concept-mapped representations of the data, and several machine learning models, using a 63,301 note corpus; in their experiments, a convolutional neural network achieved the best performance with an F1 score of 0.84. Wadekar (Wadekar, 2020) also experimented with multiple machine learning models, using 42,324 observations from the 2016 National Survey on Drug Use and Health, along with patient demographic, vital, and health information. The best model was a random forest, achieving a specificity (i.e., recall) of 0.81 and sensitivity of 0.82 (F1 was not disclosed). Prieto et al. used a document term frequency matrix derived from a corpus of 1166 paramedic trip reports containing keywords 'heroin' or naloxone', or both. Of several models tested, a logistic regression model achieved an F1 score of 0.93, based on reported data (Prieto et al., 2020).

The Veterans Health Administration (VHA) within the Department of Veterans Affairs (VA), a branch of the United States Federal Government, directs one of the world's largest healthcare systems, providing care to over 9 million Veterans at 1255 in-patient and out-patient facilities, throughout the 50 U.S. states, 4 U.S. territories, and the Philippines (Administration). It is divided into regional units known as Veterans Integrated Service Networks (VISNs), which in turn consist of regional service districts known as stations. A station generally consists of one or more hospitals, and other facilities such as outreach clinics, substance abuse care centers, and domiciliary care programs for homeless Veterans. The Washington DC and Baltimore VHA stations are part of the VISN 5, VA Capitol Health Care Network, consisting of 6 stations based in Washington DC, Maryland, and West Virginia. The EMR developed by the VA, called the Veterans Health Information Systems and Technology Architecture (VistA)(Brown et al., 2003), is one of the earliest developed and widely used EMRs in the world. These efforts have resulted in a vast clinical data resource, housed in the VHA's corporate data warehouse, and made available through the Veterans Affairs Informatics and Computing Infrastructure (VINCI), a secure research platform.

In this retrospective study, we sought to develop an NLP tool to identify opioid abuse and its treatment as documented in clinical notes, using a corpus of notes recorded between 2010 and 2019 that were drawn from the Washington DC and Baltimore stations. We applied NLP analysis methods in data preparation, and a subsequent support vector machine (SVM) model. To place these findings in context, we independently examined structured and unstructured data from the two stations, within the same ten-year period, to extrapolate on commonalities and differences in terms of patient demographics, prescribing patterns, and opioid use, abuse and misuse rates. All data retrieval, and NLP and machine learning processes were performed within the VINCI platform. This project was carried out in support of the VA Clinical NLP Ecosystem study, which was approved by the VA Central IRB \#CIRB 13-17 Zeng. No patients were contacted, only data from the EMR were used. 


\section{METHODS}

\subsection{Data for NLP and Machine Learning}

We extracted 495 clinical notes for patients in the Washington DC and Baltimore VA stations that contained key phrases associated with opioid and other forms of substance abuse. A committee of clinicians familiar with OUD documentation in clinical notes in the VA identified these phrases. The key phrases were "opioid", "opioids", “dependence", "addiction", “detox", "withdrawal", "taper”, and "tapered". We then extracted snippets, i.e. segments of text including the key phrase used to retrieve the given note, within a contextual window of the 15 terms preceding the key phrase, and the 15 terms following it in the note. One author labeled each snippet with one of three categories: 1) no evidence of opioid abuse, 2) evidence of opioid abuse without treatment, and 3) evidence of opioid abuse with treatment. To validate this process, a master set of 50 randomly selected snippets was drawn, and then reviewed by a second author. Where there were differences in labeling, the two annotators reached a consensus and made changes in the classification of the full body of snippets as dictated.

After converting text to lower case, removing punctuation and common markup such as tabs, carriage returns, and excessive whitespace, and removing non-alphanumeric terms, we identified bigrams that occurred more than four times throughout the corpus. The features for each clinical note were the occurrence frequencies of each bigram within the note, and the character offset at which the key phrase used to retrieve the note occurred within its content. The data, consisting of these features for each note, were divided into training $(80 \%)$ and test $(20 \%)$ sets.

\subsection{SVM}

The SVM implemented a radial basis function (RBF) kernel, regularization parameter C of 1.0, scaled gamma, a stopping criterion tolerance of 1e-3, and the one-vs-rest decision function shape (all default values for the RBF kernel). We used the python scikit-learn svm SVC package. The SVM model was trained using $80 \%$ of the data, and tested using the other $20 \%$ that had been reserved for that purpose. There were 137, 53, and 80 support vectors for the respective classes no evidence of opioid abuse, evidence of opioid abuse without treatment, and evidence of opioid abuse with treatment. We evaluated the model's performance for precision (true positives/(true positives + false positives)), recall (true positives/(true positives + false negatives)), F1 score $(2 *(($ precision $*$ recall $) /($ precision + recall $)))$, and total accuracy.

\subsection{Descriptive Data Extraction}

To establish context for the extent of opioid abuse within these two VA stations, we analyzed structured and unstructured data in terms of opioid prescriptions issued between 2010 and 2019 (retrieval variables in Table 1). We extracted all prescriptions for eight opioid drugs, using their generic names as recorded in structured VA data, issued the same day as an outpatient visit at the Washington DC or Baltimore station. We then extracted counts of opioid abuse diagnoses, using ICD-9-CM and ICD-10-CM codes, where a diagnosis was recorded sometime in the study period after a prescription was issued. Because structured data is sometimes incomplete, we also searched for term patterns explicitly indicating the abuse of opioid drugs in notes recorded after receiving an opioid prescription the day of an outpatient visit, for patients who did not have a subsequent ICD-9-CM or ICD-10-CM opioid abuse diagnosis. Since notes are not limited to generic names, we also searched for common trade names of these drugs. These term patterns consisted of "opioid abuse", "opiate abuse", "abusing opiates", "abusing opioids", and the terms "abuses" and "abusing" directly preceding a specific drug (e.g., "abuses hydrocodone", "abusing oxycontin"). To gauge the utility of this last step, we reviewed 100 randomly selected notes that contained the term patterns. Of the 100 notes, 77 provided clear evidence that the associated patient was experiencing current or past opioid abuse at the time note was written.

We also extracted demographic data for these patients, including age (at time of prescription), marital status, gender, race, and ethnicity. We also extracted co-diagnosis information for traumatic brain injury, mental health issues, and pain diagnosis. 
Table 1. Structured and unstructured data retrieval variables

\begin{tabular}{|c|c|c|}
\hline Structured Data & Type & Example Values \\
\hline Drug & Categorical & $\begin{array}{l}\text { Morphine, Codeine, Tramadol, Hydrocodone, Oxycodone, } \\
\text { Hydromorphone, Oxymorphone, Fentanyl (Patch or Lozenge) }\end{array}$ \\
\hline Patient & Numeric \& & Age, Gender, Ethnicity, Race, Marital Status \\
\hline Demographics & Categorical & \\
\hline $\begin{array}{l}\text { OUD Diagnosis } \\
\text { (outcome variable) }\end{array}$ & Binary & $\begin{array}{l}\text { ICD-9-CM codes }(304.0,304.00-304.03,304.7,304.70-304.73 \text {, } \\
\text { 305.50-305.53, 965.00, E850.2, E935.2); ICD-10 codes (F11.10- } \\
\text { F11.19, F11.20 - F11.29); }\end{array}$ \\
\hline Pain Diagnosis & Binary & $\begin{array}{l}\text { ICD-9-CM codes (715.16, 715.96, 719.40-43, 719.45-49, 723.1, } \\
\text { 724.2, 724.4, 729.5, 524.60-64, 524.69); ICD-10 codes (G43-, G44-, } \\
\text { G50-, M25.5, M26.6, M54-, M79.1-2, M79.5-7, R51-); }\end{array}$ \\
\hline TBI Diagnosis & Binary & $\begin{array}{l}\text { ICD-9-CM codes (310.2, 800-, 801-, 803-, 804-, 850-, 852-, 853-, } \\
854-, \text { 907.0, 959,01); ICD-10 codes (F07.81, S01-, S02-, S06-, } \\
\text { S09.8, Z87.820); }\end{array}$ \\
\hline $\begin{array}{l}\text { Mental Health } \\
\text { Diagnosis }\end{array}$ & Binary & $\begin{array}{l}\text { ICD-9-CM codes (296.2-3, 300.00-02, 300.09, 309.81-82, 309.89, } \\
\text { 311-); ICD-10 codes (F32-, F33, F41-, F43-); }\end{array}$ \\
\hline $\begin{array}{l}\text { Unstructured } \\
\text { Data }\end{array}$ & Type & Example Values \\
\hline Pattern phrases & Binary & $\begin{array}{l}\text { "opioid abuse" "abusing oxycodone" "abuses oxycontin"; generic } \\
\text { drug names used in structured data; additional trade names: } \\
\text { Oxycontin, Lortab, Percodan, Percocet, Vicodin, MS Contin, } \\
\text { Roxanl, Dilaudid }\end{array}$ \\
\hline
\end{tabular}

\section{RESULTS}

\subsection{SVM}

The SVM achieved $92.9 \%$ accuracy, with weighted averages of $93 \%$ for precision, recall, and F1 score (Table 2). The SVM, with the hyperparameters described in the methods section, achieved this performance using a relatively small corpus of clinical notes.

Table 2. SVM results

\begin{tabular}{lllll}
\hline Class & Precision & Recall & F1 & Support (Class Occurrence) \\
\hline 1: no evidence of opioid abuse & 0.90 & 1.00 & 0.95 & 55 \\
2: evidence of opioid abuse without treatment & 0.92 & 0.85 & 0.88 & 13 \\
3: evidence of opioid abuse with treatment & 1.00 & 0.84 & 0.91 & 31 \\
\hline Weighted on Support/Total Support & 0.93 & 0.93 & 0.93 & \\
\hline
\end{tabular}

\subsection{Descriptive Data Analysis}

Within the study period a total of 609777 prescriptions were issued to 69398 unique patients, as itemized in Table 3. Because many patients received prescriptions for multiple years, there is a difference between the cumulative patient counts and the sums of the year-by-year patient counts. Calculating the cumulative patient count (i.e., unique patients served in the ten-year period) provides an overview of the patient population receiving opioid prescriptions during the study. Over the ten-year period, 69398 patients received opioid prescriptions, with an average of 8.8 prescriptions per patient, and an average of 768 days between the first and last prescription. 
Table 3. Descriptive data analysis, prescriptions issued and patients receiving prescriptions during the study period

\begin{tabular}{|c|c|c|c|c|c|c|}
\hline & $\begin{array}{l}\text { All } \\
\text { Prescriptions }\end{array}$ & All Patients & $\begin{array}{l}\text { DCVA } \\
\text { Prescriptions }\end{array}$ & $\begin{array}{l}\text { DCVA } \\
\text { Patients }\end{array}$ & $\begin{array}{l}\text { Baltimore } \\
\text { Prescriptions }\end{array}$ & $\begin{array}{l}\text { Baltimore } \\
\text { Patients }\end{array}$ \\
\hline $\begin{array}{l}\text { Cumulative } \\
\text { (unique to study } \\
\text { period) }\end{array}$ & 609774 & 69398 & 287553 & 35353 & 322221 & 34045 \\
\hline 2010 & 66834 & 17981 & 32394 & 8935 & 34440 & 9046 \\
\hline 2011 & 73159 & 19356 & 35202 & 9530 & 37957 & 9826 \\
\hline 2012 & 74434 & 19758 & 35436 & 9624 & 38998 & 10134 \\
\hline 2013 & 75079 & 19984 & 35915 & 9768 & 39164 & 10216 \\
\hline 2014 & 74658 & 20101 & 34613 & 9751 & 40045 & 10350 \\
\hline 2015 & 69780 & 19058 & 31919 & 9318 & 37861 & 9740 \\
\hline 2016 & 60660 & 16382 & 26915 & 7940 & 33745 & 8442 \\
\hline 2017 & 48532 & 13796 & 21876 & 6701 & 26656 & 7095 \\
\hline 2018 & 37382 & 11067 & 17953 & 5512 & 19429 & 5555 \\
\hline 2019 & 29256 & 9187 & 15330 & 4837 & 13926 & 4350 \\
\hline
\end{tabular}

Of the 69398 patients receiving at least one opioid prescription, 4209 received at least one opioid abuse diagnosis (by ICD code) after receiving a prescription. These patients received an average of 22.3 opioid prescriptions, with an average of 1388 days between the first and last prescription. After applying the NLP tool to the clinical notes of the patients receiving prescriptions but not an opioid diagnosis, we identified 809 additional patients with opioid abuse documented in their clinical notes within the study's time period. Table 4 itemizes these results by year and station. Again, comparing the cumulative totals to the year-by-year figures reveals that there are select patients counted for multiple years, as these patients received a prescription and a later diagnosis, or alternatively a prescription and documentation indicating opioid abuse issues, for multiple years.

Table 4. Descriptive data analysis, patients suspected of experiencing OUD; findings presented side-by-side by data analysis type

\begin{tabular}{lrrrrrr}
\hline & \multicolumn{3}{l}{ Structured Data Analysis } & \multicolumn{5}{l}{ Clinical Notes Analysis } \\
\hline & $\begin{array}{l}\text { Both } \\
\text { Stations }\end{array}$ & DCVA & Baltimore & $\begin{array}{l}\text { Both } \\
\text { Stations }\end{array}$ & DCVA & Baltimore \\
Cumulative (unique & & & & & & \\
to study period) & 4209 & 1758 & 2451 & 809 & 203 & 606 \\
2010 & 676 & 234 & 442 & 61 & 18 & 43 \\
2011 & 985 & 352 & 633 & 87 & 23 & 64 \\
2012 & 1241 & 416 & 825 & 111 & 29 & 82 \\
2013 & 1344 & 474 & 870 & 135 & 34 & 101 \\
2014 & 1307 & 492 & 815 & 118 & 23 & 95 \\
2015 & 1321 & 491 & 830 & 139 & 36 & 103 \\
2016 & 1152 & 439 & 713 & 136 & 31 & 105 \\
2017 & 1181 & 430 & 751 & 141 & 34 & 107 \\
2018 & 1190 & 385 & 805 & 91 & 20 & 71 \\
2019 & 1145 & 344 & 801 & 121 & 32 & 89 \\
\hline
\end{tabular}

There are notable trends within these results, by station and by year. While the cumulative number of patients receiving prescriptions over the study period by station is comparable (slightly more for Washington DC than Baltimore) more prescriptions were issued by the Baltimore station; however, the decrease in prescriptions issued in the last three years is especially notable in the Baltimore data (Table 3). In addition, there are more patients in the Baltimore data suspected of experiencing OUD, in both the structured data and clinical notes analyses (Table 4).

The patient demographic and co-diagnosis data (Table 5) are itemized according to percentage and data type. Data points are separated by data type groups, including gender, race, ethnicity, marital status, and co-diagnosis. 
Table 5. Patient demographic and co-diagnosis percentages, by data type

\begin{tabular}{|c|c|c|}
\hline Data Point & Structured Data & Clinical Notes \\
\hline \multicolumn{3}{|l|}{ Patient Demographics } \\
\hline Male & $92.8 \%$ & $90 \%$ \\
\hline Female & $7.2 \%$ & $10 \%$ \\
\hline Mean Age & 55.1 years & 57.8 years \\
\hline \multicolumn{3}{|l|}{ Patient Race } \\
\hline Black or African American & $67.6 \%$ & $58.6 \%$ \\
\hline White & $29.5 \%$ & $37.3 \%$ \\
\hline Declined to Answer & $1 \%$ & $1.1 \%$ \\
\hline Native Hawaiian or Pacific Islander & $<1 \%$ & $<1 \%$ \\
\hline Unknown by Patient & $<1 \%$ & $<1 \%$ \\
\hline Asian & $<1 \%$ & $<1 \%$ \\
\hline American Indian or Alaska Native & $<1 \%$ & $<1 \%$ \\
\hline \multicolumn{3}{|l|}{ Ethnicity } \\
\hline Not Hispanic or Latino & $97.8 \%$ & $96.4 \%$ \\
\hline Hispanic or Latino & $1.3 \%$ & $1.2 \%$ \\
\hline Unknown by Patient & $<1 \%$ & $1.4 \%$ \\
\hline Declined to Answer & $<1 \%$ & $1 \%$ \\
\hline \multicolumn{3}{|l|}{ Marital Status } \\
\hline Married & $24.7 \%$ & $22.6 \%$ \\
\hline Divorced & $32.7 \%$ & $37.2 \%$ \\
\hline Never Married & $25.5 \%$ & $25.3 \%$ \\
\hline Separated & $12.7 \%$ & $10 \%$ \\
\hline Widowed & $4.4 \%$ & $4.7 \%$ \\
\hline Unknown & $<1 \%$ & $<1 \%$ \\
\hline \multicolumn{3}{|l|}{ Co-diagnosis } \\
\hline Traumatic Brain Injury (ICD-9-CM / ICD-10-CM) & $5.7 \% / 5.7 \%$ & $5.4 \% / 6.1 \%$ \\
\hline Mental Health Issues (ICD-9-CM / ICD-10-CM) & $47.4 \% / 58.8 \%$ & $44.4 \% / 57.5 \%$ \\
\hline Pain (ICD-9-CM / ICD-10-CM) & $82.6 \% / 68.4 \%$ & $79.6 \% / 65.1 \%$ \\
\hline
\end{tabular}

Percentage breakdowns for the demographic data are inclusive by data type group, and differences are due to rounding. In the co-diagnosis data, percentages are indicative of the number of diagnoses for unique patients, for the given co-diagnosis in terms of the primary diagnosis of OUD, according to ICD code type.

\section{DISCUSSION}

Empirically, clinicians and clinical researchers believe that the ICD coding of opioid abuse does not capture all known cases. This study demonstrates that NLP and machine learning methods can identify additional patients who are potentially experiencing opioid related problems, (with and without treatment), and differentiate them from those experiencing other forms of substance abuse, using clinical notes. Many of the notes classified as showing no evidence of opioid abuse did document other forms of substance abuse. In our experiment, the NLP tool identified over 800 patients who did not have ICD codes for OUD (4209 patients had been identified by ICD codes). F1 scores of 0.88 and 0.91 for evidence of opioid abuse without treatment and with treatment, respectively, demonstrate that these types of documents (and the associated patients) can be identified with considerable exactitude, even using a relatively small corpus. The weighted averages of 0.93 for precision, recall, and F1 are comparable to the similar projects described in the Introduction, but through leveraging less data. Thus, healthcare institutions and researchers can identify patients potentially suffering from opioid abuse through clinical note analysis, in addition to coded data, with less annotation burden.

The descriptive data analysis demonstrates geographical differences in terms of opioid prescriptions and opioid abuse. More prescriptions were issued by the Baltimore station, despite there being slightly more cumulative patients served by the Washington DC station during the study period (Table 3). More Baltimore station patients exhibited evidence of opioid abuse than did those of the Washington DC station, particularly in the analysis of clinical notes (Table 4). The dynamics driving these effects warrant further study.

There is notable variation in the number of opioid prescriptions issued by year (Table 3). These relate to some extent to the policies and practice guidelines outlined in the Introduction, suggesting that these measures have had a positive effect in reducing overall prescription frequencies. In contrast, we do not see the same 
effect in OUD (Table 4). The study's cumulative patient counts (Table 3) for each station are comparable, and their surprisingly small numbers (35353 for Washington DC and 34045 for Baltimore) compared to the amount of prescriptions issued over the ten-year study period, and the amount of patients receiving a prescription each year, suggest that opioid use, abuse and misuse are ongoing issues for many Veterans.

There is notable variation within the OUD patient demographic data (Table 5), in terms of comparing OUD documentation in structured and unstructured data. This is especially seen in regard to race, and to a lesser extent in co-diagnosis. In the co-diagnosis breakdowns, percentages represent the total of OUD patients receiving a specific type of co-diagnosis code, which depends on year. The VA phased out ICD-9-CM and began using ICD-10-CM in 2015, which was the sixth year of this ten-year retrospective study. While these percentages are derived using the OUD totals for the ten-year study, they are comparable to findings in a similar 2012-2018 study (Workman et al., 2019b), thus further demonstrating that OUD is a problem framed by an extended, multiyear viewpoint. Also, the differences in average prescriptions (8.8 vs. 22.3) and average days between first and last prescription (768 vs. 1388) between all patients and those receiving an OUD diagnosis reveal OUD is an issue framed by time as well as drug quantity. In this current study, the differences in percentage, by type of code, suggest tendencies for clinicians to note OUD for patients with the given co-diagnosis over time. They suggest that clinicians are more likely to note OUD, in both structured and unstructured mediums, for patients experiencing traumatic brain injury and mental health issues after 2015, and less likely to do so for patients with pain diagnoses. The collective reasons behind these differences also warrant further analysis.

\subsection{Future Work}

The future work inspired by this study addresses additional efforts in machine learning-enabled clinical note identification, and data analysis. We intend to develop NLP and machine learning methods to distinguish notes documenting OUD by station. This can be seen as a sublanguage task (Doing-Harris et al., 2013, Workman et al., 2019a, Zeng et al., 2011) benefiting from sublanguage-oriented techniques. We also intend to further study the dynamics behind the differences in data, in terms of station prescription issuance, and demographic and co-diagnosis data, particularly in terms of structured and unstructured data types.

\section{CONCLUSION}

We developed an NLP tool to identify instances of opioid abuse receiving treatment, and instances of opioid abuse not receiving treatment, among Veterans served by two VA stations, using a corpus of clinical notes. The tool achieved significant performance, demonstrating that opioid abuse can be detected by healthcare institutions through NLP and machine learning, even using a small corpus. To better understand the results, we also retrieved and analyzed data for opioid prescriptions, opioid abuse, patient demographics, and co-diagnoses, from structured and unstructured data (i.e., clinical notes). This resulted in notable trends and differences among the two VA stations.

\section{ACKNOWLEDGEMENT}

The views expressed are those of the authors and do not necessarily reflect those of the Department of Veterans Affairs, the United States Government, or the academic affiliate organizations. This work was supported by VA Projects SIP 18-329 Utilization of Opioids in Post 9/11 Veterans in the VA Community, HSR\&D CRE 12-315 A VHA NLP Software Ecosystem for Collaborative Development and Integration, and NIH grant UL1TR001876 from the National Center for Advancing Translational Sciences.

\section{REFERENCES}

Administration, V. H. About VA [Online]. Available: https://www.va.gov/about_va/ [Accessed 04 May 2020].

Association, A. P. 2013. Diagnostic and statistical manual of mental disorders (DSM-5®), American Psychiatric Pub.

Brown, S. H., Lincoln, M. J., Groen, P. J. \& Kolodner, R. M. 2003. VistA--U.S. Department of Veterans Affairs National-scale HIS. Int J Med Inform, 69, 135-56. 
Chartoff, E. H. \& Mavrikaki, M. 2015. Sex Differences in Kappa Opioid Receptor Function and Their Potential Impact on Addiction. Front Neurosci, 9, 466.

Cottler, L. B., Ben Abdallah, A., Cummings, S. M., Barr, J., Banks, R. \& Forchheimer, R. 2011. Injury, Pain, and Prescription Opioid Use among Former National Football League (NFL) Players. Drug Alcohol Depend, 116, $188-94$.

Doing-Harris, K., Patterson, O., Igo, S. \& Hurdle, J. 2013. Document Sublanguage Clustering to Detect Medical Specialty in Cross-institutional Clinical Texts. Proc ACM Int Workshop Data Text Min Biomed Inform, 2013, 9-12.

Edlund, M. J., Steffick, D., Hudson, T., Harris, K. M. \& Sullivan, M. 2007. Risk Factors for Clinically Recognized Opioid Abuse and Dependence among Veterans Using Opioids for Chronic Non-cancer Pain. Pain, 129, 355-62.

Garcia, M. C., Heilig, C. M., Lee, S. H., Faul, M., Guy, G., Iademarco, M. F., Hempstead, K., Raymond, D. \& Gray, J. 2019. Opioid Prescribing Rates in Nonmetropolitan and Metropolitan Counties Among Primary Care Providers Using an Electronic Health Record System - United States, 2014-2017. MMWR Morb Mortal Wkly Rep, 68, 25-30.

Gellad, W. F., Good, C. B. \& Shulkin, D. J. 2017. Addressing the Opioid Epidemic in the United States: Lessons From the Department of Veterans Affairs. JAMA Intern Med, 177, 611-612.

Green, C. R., Anderson, K. O., Baker, T. A., Campbell, L. C., Decker, S., Fillingim, R. B., Kalauokalani, D. A., Lasch, K. E., Myers, C., Tait, R. C., Todd, K. H. \& Vallerand, A. H. 2003. The Unequal Burden of Pain: Confronting Racial and Ethnic Disparities in Pain. Pain Med, 4, 277-94.

Hastings, J. S., Howison, M. \& Inman, S. E. 2020. Predicting High-risk Opioid Prescriptions before they are Given. Proc Natl Acad Sci U S A, 117, 1917-1923.

Kendler, K. S., Ohlsson, H., Sundquist, K. \& Sundquist, J. 2013. A Latent Class Analysis of Drug Abuse in a National Swedish Sample. Psychol Med, 43, 2169-78.

Lin, L. A., Bohnert, A. S. B., Kerns, R. D., Clay, M. A., Ganoczy, D. \& Ilgen, M. A. 2017. Impact of the Opioid Safety Initiative on Opioid-related Prescribing in Veterans. Pain, 158, 833-839.

Lingeman, J. M., Wang, P., Becker, W. \& Yu, H. 2017. Detecting Opioid-Related Aberrant Behavior using Natural Language Processing. AMIA Annu Symp Proc, 2017, 1179-1185.

Management, V. P. Opioid Safety Initiative (OSI) [Online]. Available: https://www.va.gov/painmanagement/opioid_safety_initiative_osi.asp [Accessed 04 May 2020].

Manubay, J., Davidson, J., Vosburg, S., Jones, J., Comer, S. \& Sullivan, M. 2015. Sex Differences among Opioid-abusing Patients with Chronic Pain in a Clinical Trial. J Addict Med, 9, 46-52.

Martins, S. S., Sampson, L., Cerda, M. \& Galea, S. 2015. Worldwide Prevalence and Trends in Unintentional Drug Overdose: A Systematic Review of the Literature. Am J Public Health, 105, e29-49.

Moss, J., Andison, M. \& Sobko, H. 2007. An Analysis of Narrative Nursing Documentation in an otherwise Structured Intensive Care Clinical Information System. AMIA Annu Symp Proc, 543-7.

Prieto, J. T., Scott, K., Mcewen, D., Podewils, L. J., Al-Tayyib, A., Robinson, J., Edwards, D., Foldy, S., Shlay, J. C. \& Davidson, A. J. 2020. The Detection of Opioid Misuse and Heroin Use From Paramedic Response Documentation: Machine Learning for Improved Surveillance. J Med Internet Res, 22, e15645.

Sarker, A., Gonzalez-Hernandez, G. \& Perrone, J. 2019a. Towards Automating Location-Specific Opioid Toxicosurveillance from Twitter via Data Science Methods. Stud Health Technol Inform, 264, 333-337.

Sarker, A., Gonzalez-Hernandez, G., Ruan, Y. \& Perrone, J. 2019b. Machine Learning and Natural Language Processing for Geolocation-Centric Monitoring and Characterization of Opioid-Related Social Media Chatter. JAMA Netw Open, 2, e1914672.

Scholl, L., Seth, P., Kariisa, M., Wilson, N. \& Baldwin, G. 2018. Drug and Opioid-Involved Overdose Deaths - United States, 2013-2017. MMWR Morb Mortal Wkly Rep, 67, 1419-1427.

Sharma, B., Dligach, D., Swope, K., Salisbury-Afshar, E., Karnik, N. S., Joyce, C. \& Afshar, M. 2020. Publicly Available Machine Learning Models for Identifying Opioid Misuse from the Clinical Notes of Hospitalized Patients. BMC Med Inform Decis Mak, 20, 79.

Wadekar, A. S. 2020. Understanding Opioid Use Disorder (OUD) Using Tree-based Classifiers. Drug Alcohol Depend, 208, 107839.

Webster, L. R. 2017. Risk Factors for Opioid-Use Disorder and Overdose. Anesth Analg, 125, 1741-1748.

Wentling, N. 2018. Watchdog: VA Cuts Opioid Prescription Rate by 41 Percent in Past 5 Years. Stars and Stripes.

Workman, T. E., Divita, G. \& Zeng-Treitler, Q. Discovering Sublanguages in a Large Clinical Corpus through Unsupervised Machine Learning and Information Gain. 2019 IEEE International Conference on Big Data (Big Data), 2019a. IEEE, 4889-4898.

Workman, T. E., Shao, Y., Kupersmith, J., Sandbrink, F., Goulet, J. L., Shaar, N. M., Spevak, C., Brandt, C., Blackman, M. R. \& Zeng-Treitler, Q. Explainable Deep Learning Applied to Understanding Opioid Use Disorder and Its Risk Factors. 2019 IEEE International Conference on Big Data (Big Data), 2019b. IEEE, 4883-4888.

Zeng, Q. T., Redd, D., Divita, G., Jarad, S., Brandt, C. \& Nebeker, J. 2011. Characterizing Clinical Text and Sublanguage: A Case Study of the VA Clinical Notes. J Health Med Informat S, 3, 2. 